\title{
Effects of Thermal Radiation and Rotation on Unsteady Hydromagnetic Free Convection Flow past an Impulsively Moving Vertical Plate with Ramped Temperature in a Porous Medium
}

\author{
G.S. Seth ${ }^{1 \dagger}$, R. Nandkeolyar ${ }^{2}$ and M. S. Ansari ${ }^{3}$ \\ ${ }^{I}$ Department of Applied Mathematics, Indian School of Mines, Dhanbad-826004, India \\ ${ }^{2}$ Department of Mathematics, KIIT University, Bhubaneswar-751024, India \\ ${ }^{3}$ School of Technology, Pandit Deendayal Petroleum University, Gandhinagar-382007, India
}

$\dagger$ Corresponding Author Email: gsseth_ism@yahoo.com

(Received September 1, 2010; accepted July 10, 2011)

\begin{abstract}
The effects of radiation and rotation on unsteady hydromagnetic free convection flow of a viscous incompressible electrically conducting fluid past an impulsively moving infinite vertical plate with ramped temperature in a porous medium are investigated. Exact solution of momentum and energy equations, under Boussinesq approximation, is obtained in closed form by Laplace transform technique. To compare the results obtained in this case with that of isothermal plate, exact solution of the governing equations is also obtained for isothermal plate. The expressions for the primary and secondary skin frictions and Nusselt number are also derived. It is noticed that, for both ramped temperature and isothermal plates, rotation retards fluid flow in the primary flow direction whereas it accelerates fluid flow in the secondary flow direction in the boundary layer region while radiation exerts accelerating influence on the fluid flow in both the primary and secondary flow directions. For ramped temperature plate radiation reduces primary skin friction whereas it tends to increase secondary skin friction. For isothermal plate radiation has tendency to reduce secondary skin friction. Radiation tends to increase fluid temperature for both ramped temperature and isothermal plates. With the increase in time the rate of heat transfer at the plate is reduced for isothermal plate while it is increased for ramped temperature plate.
\end{abstract}

Keywords: Thermal radiation, Hydromagnetic free convection, Rotation, Ramped temperature, Porous medium

\section{INTRODUCTION}

An investigation of convective heat transfer from a solid body with different geometries embedded in a fluid saturated porous medium has varied and wide applications in many areas of science and engineering such as geothermal reservoirs, drying of porous solids, chemical catalytic reactors, thermal insulators, nuclear waste repositories, heat exchanger devices, enhanced oil and gas recovery, underground energy transport etc. Keeping in view this fact, Cheng and Minkowycz (1977) obtained similarity solution for free convection flow from a vertical plate embedded in a fluid saturated medium. Ranganathan and Viskanta (1984) considered mixed convection boundary layer flow along a vertical surface in a porous medium whereas Minkowycz et al. (1985) analyzed the effects of mixed convection about a non-isothermal cylinder and sphere in a porous medium. Nakayama and Koyama (1987) investigated combined free and forced convection flows in Darcian and non-Darcian porous media. Hsieh et al. (1993) found non-similar solutions for mixed convection from vertical surfaces in a porous medium. Lai and Kulacki (1991) considered coupled heat and mass transfer by mixed convection from an isothermal vertical wall in a saturated porous medium. Comprehensive reviews of porous media thermal/species convection are well presented by (Nield and Bejan 2006; Vafai 2005; Pop and Ingham 2002).

The problems of magnetohydrodynamic flow and heat transfer in porous and non-porous media have drawn attention of many researchers due to the significant effects of magnetic field on the boundary layer flow control and on the performance of many systems using electrically conducting fluid. In addition, this type of fluid flow finds application in MHD power generation, MHD pumps, flow meters and accelerators, plasma studies, nuclear reactors using liquid metal coolants and geothermal energy extraction. Raptis and Kafousias (1982) studied the effect of magnetic field on steady free convection flow through a porous medium bounded by an infinite vertical plate with constant suction velocity. Raptis (1986) investigated unsteady two dimensional natural convection flow of an incompressible electrically conducting fluid along an infinite vertical porous plate embedded in a porous medium. Sahoo and Sahoo (1986) analyzed hydromagnetic free convection and mass transfer flow past an impulsively moving vertical plate through porous 
medium while Jha (1991) considered this problem for uniformly accelerated movement of the vertical plate. Aldoss et al. (1995) studied mixed convection flow from a vertical plate embedded in a porous medium in the presence of magnetic field. Takhar et al. (1991) analyzed the effects of Hall current on MHD free convection boundary layer flow via a porous medium past a plate with wall temperature oscillations using harmonic analysis. Takhar et al. (1994) also studied the MHD free convection flow of water at $4^{\circ} \mathrm{C}$ through a porous medium. Helmy (1998) considered unsteady two dimensional laminar free convection flow of an incompressible, electrically conducting fluid (Newtonian or polar) through a porous medium bounded by infinite vertical plane surface of constant temperature. Kim (2000) investigated an unsteady MHD convection flow past a vertical moving plate embedded in a porous medium. Beg et al (2001) presented a two dimensional computational fluid dynamics analysis of steady thermal boundary layer flow of a second order non-Newtonian fluid past a horizontal wedge in a Brinkman-Darcy porous medium. Ibrahim et al. (2004) considered unsteady MHD micro polar fluid flow and heat transfer over a vertical porous plate through a porous medium in the presence of thermal and mass diffusion with a constant heat source.

In all these investigations the effects of radiation is not taken into account. However, in some industrial applications such as glass production, furnace design, thermonuclear fusion, casting and levitation and in space technology applications such as cosmical flight, propulsion systems, plasma physics, and space re-entry aerodynamics which operate at higher temperature, radiation effects become significant. Keeping in view this fact, Takhar et al. (1996) investigated the effects of radiation on MHD free convection flow of a gas past a semiinfinite vertical plate whereas Hossain et al. (1996) analyzed the effects of radiation on mixed convection along a vertical plate with uniform temperature. Bakier et al. (1996) considered the effect of radiation on mixed convection flow over a horizontal surface embedded in a saturated porous medium. Chamkha (2000) investigated thermal radiation and buoyancy effects on MHD flow over an accelerating permeable surface with heat source or sink. Azzam (2002) considered radiation effects on MHD mixed free and forced convection flow past a semi infinite moving vertical plate for high temperature differences. Israel-Cookey et al. (2003) studied the influence of viscous dissipation and radiation on unsteady MHD free convection flow past an infinite heated vertical plate in a porous medium with time-dependent suction. Kim and Fedorov (2003) considered the transient mixed radiative convection flow of a micro polar fluid past a moving semi-infinite vertical porous plate. Prasad et al. (2007) investigated unsteady, laminar, simultaneous free convective heat and mass transfer flow along an impulsively started plate in the presence of radiation effects. Mahmoud Mostafa (2009) considered thermal radiation effect on unsteady MHD free convection flow of an electrically conducting fluid past an infinite vertical porous plate taking into account the effects of viscous dissipation.

The problems of hydromagnetic free convection flow in a rotating medium taking into account the effects of radiation are of considerable importance in many areas of geophysics, astrophysics and fluid engineering. Keeping in view this fact,
Bestman et al. (1998) investigated unsteady hydromagnetic free convection flow of an incompressible optically thick fluid with radiative heat transfer near a moving flat plate in a rotating medium by imposing a time dependent perturbation on a constant plate temperature. Mbeledogu et al. (2007) considered heat and mass transfer of an unsteady MHD natural convection flow of a rotating fluid past a vertical porous plate in the presence of radiative heat transfer. They applied Rosseland approximation for an optically thick fluid to describe the radiative flux.

In all these investigations, the analytical or numerical solution is obtained assuming velocity and temperature conditions at the plate as continuous and well defined. However, several practical problems may require non-uniform or arbitrary wall conditions. Taking into account this fact, several researchers (Haday et al. 1967; Kelleher 1971; Kao 1975; Lee and Yovanovich (1991) considered the problems of free convection from a vertical plate with step discontinuities in the surface temperature. Recently Chandran et al. (2005) investigated unsteady natural convection flow of a viscous incompressible fluid near a vertical plate with ramped wall temperature. They compared the results of natural convection near a ramped temperature plate with that of natural convection near an isothermal plate.

The present study deals with the study of the effects of radiation and rotation on unsteady hydromagnetic free convection flow of a viscous incompressible electrically conducting fluid past an impulsively moving vertical plate in a porous medium, under Boussinesq approximation, assuming that the temperature of the plate has a temporarily ramped profile. Free convection resulting from such a plate temperature profile is likely to be of relevance in many engineering applications, especially where the initial temperature profiles assume importance in designing of MHD devices and in several natural phenomena occurring due to convection and radiation. An exact solution of the governing equations, in dimensionless form, is obtained by Laplace transform technique. To compare the results obtained in this case with that of isothermal plate, exact solution of the governing equations is also obtained for isothermal plate. The expressions for the primary and secondary skin frictions and Nusselt number are also derived for both ramped temperature and isothermal plates. Mathematical formulation of the problem, in dimensionless form, contains six pertinent flow parameters, namely, $M$ (magnetic parameter), $K^{2}$ (rotation parameter which is reciprocal of Ekman number), $G_{r}$ (Grashof number), $K_{1}$ (porosity parameter), $P_{r}$ (Prandtl number) and $N$ (radiation parameter). The numerical values of the primary and secondary velocities are displayed graphically versus boundary layer coordinate $\eta$ for various

values of $M, K^{2}, G_{r}, K_{1}, N$ and $t$ while the profiles of fluid temperature are drawn versus $\eta$ for various values of $P_{r}, N$ and $t$ for both ramped temperature and isothermal plates. The numerical values of the primary and secondary skin frictions are presented in tables for various values of $M$, $K^{2}, G_{r}, K_{1}, N$ and $t$ whereas that of Nusselt number are 
provided in tabular form for different values of $N, P_{r}$ and $t$ for both ramped temperature and isothermal plates.

\section{Formulation of The Problem AND it'S SOLUTION}

Consider unsteady hydromagnetic free convection flow of a viscous incompressible electrically conducting fluid past an impulsively moving infinite vertical plate embedded in a porous medium. Choose the coordinate system in such a way that the $x$-axis is taken along the plate in the upward direction, $y$-axis normal to the plane of the plate in the fluid and z-axis perpendicular to $x y$ - plane. The fluid is permeated by a uniform transverse magnetic field $B_{0}$ applied parallel to $y$ axis. Both the fluid and plate rotate in unison with a uniform angular velocity $\Omega$ about $y$-axis. Initially, at time $t^{\prime} \leq 0$, both the fluid and plate are at rest and at a constant temperature $T_{\infty}^{\prime}$. At time $t^{\prime}>0$, the plate starts moving in $x$ direction with uniform velocity $U_{0}$ and the temperature of the plate is raised or lowered to $T_{\infty}^{\prime}+\left(T_{w}^{\prime}-T_{\infty}^{\prime}\right) t^{\prime} / t_{0}$ when $t^{\prime} \leq_{0}$, and thereafter, for $t^{\prime}>t_{0}$, is maintained at the constant temperature $T_{w}^{\prime}$. Since the plate is infinite in $x$ and $z$ directions and is electrically non-conducting all physical quantities, except pressure, will be functions of $y$ and $t^{\prime}$ only. Therefore, the fluid velocity $\vec{q}$ and magnetic field $\vec{B}$ is given by

$\vec{q} \equiv\left(u^{\prime}, 0, w^{\prime}\right), \vec{B} \equiv\left(B_{x}, B_{0}, B_{z}\right)$,

which is compatible with the fundamental equations of magnetohydrodynamics in a rotating frame of reference. It is assumed that the induced magnetic field produced by the fluid motion is negligible in comparison to the applied one so that we consider magnetic field $\vec{B} \equiv\left(0, B_{0}, 0\right)$. This assumption is justified because magnetic Reynolds number is very small for metallic liquids and partially ionized fluids (Cramer and Pai (1973)). Also no external electric field is applied so the effects of polarization of fluid is negligible (Meyer (1958)), i.e. electric field $\vec{E} \equiv(0,0,0)$.

Taking into consideration the assumptions made above, the governing equations for laminar free convection flow of a viscous incompressible electrically conducting fluid past a vertical plate in a uniform porous medium with radiative heat transfer, under Boussinesq approximation, in a rotating frame of reference are

$$
\begin{aligned}
& \frac{\partial u^{\prime}}{\partial t^{\prime}}+2 \Omega w^{\prime}=v \frac{\partial^{2} u^{\prime}}{\partial y^{2}}-\frac{\sigma B_{0}^{2}}{\rho} u^{\prime}-\frac{v}{K_{1}^{\prime}} u^{\prime}+g \beta^{\prime}\left(T^{\prime}-T_{\infty}^{\prime}\right), \\
& \frac{\partial w^{\prime}}{\partial t^{\prime}}-2 \Omega u^{\prime}=v \frac{\partial^{2} w^{\prime}}{\partial y^{2}}-\frac{\sigma B_{0}^{2}}{\rho} w^{\prime}-\frac{v}{K_{1}^{\prime}} w^{\prime}, \\
& \frac{\partial T^{\prime}}{\partial t^{\prime}}=\frac{k}{\rho c p} \frac{\partial^{2} T^{\prime}}{\partial y^{2}}-\frac{1}{\rho c_{p}} \frac{\partial q_{r}^{\prime}}{\partial y},
\end{aligned}
$$

where $T^{\prime}, g, \beta^{\prime}, v, \sigma, \rho, k, K_{1}^{\prime}, c_{p}$ and $q_{r}^{\prime}$ are, respectively, temperature of the fluid, acceleration due to gravity, volumetric coefficient of thermal expansion, kinematic coefficient of viscosity, electrical conductivity, fluid density, thermal conductivity, permeability of porous medium, specific heat at constant pressure and radiative flux vector.

The initial and boundary conditions are

$u^{\prime}=w^{\prime}=0, T^{\prime}=T_{\infty}^{\prime}$ for $y \geq 0$ and $t^{\prime} \leq 0$,

$u^{\prime}=U_{0}, w^{\prime}=0$ at $y=0$ for $t^{\prime}>0$,

$T^{\prime}=T_{\infty}^{\prime}+\left(T_{w}^{\prime}-T_{\infty}^{\prime}\right) t^{\prime} / t_{0}$ at $y=0$ for $0<t^{\prime} \leq t_{0}$,

$T^{\prime}=T_{w}^{\prime}$ at $y=0$ for $t^{\prime}>t_{0}$,

$u^{\prime} \rightarrow 0, w^{\prime} \rightarrow 0, T^{\prime} \rightarrow T_{\infty}^{\prime}$ as $y \rightarrow \infty$ for $t^{\prime}>0$.

We now use Rosseland approximation Brewster (1992) which leads to the value of radiative heat flux $q_{r}^{\prime}$ as

$q_{r}^{\prime}=-\frac{4 \sigma^{*}}{3 k^{*}} \frac{\partial T^{\prime 4}}{\partial y}$

where $k^{*}$ is mean absorption coefficient and $\sigma^{*}$ is StefanBoltzmann constant. It may be noted that by using Rosseland approximation we limit our analysis to optically thick fluids. Assuming small temperature differences between fluid temperature $T^{\prime}$ and free stream temperature $T_{\infty}^{\prime}$, the Eq. (6)

is linearized by expanding $T^{\prime 4}$ in Taylors series about free stream temperature $T_{\infty}^{\prime}$, which after neglecting second and higher order terms in $\left(T^{\prime}-T_{\infty}^{\prime}\right)$ takes the form

$T^{\prime 4} \cong 4 T_{\infty}^{\prime 3} T^{\prime}-3 T_{\infty}^{\prime 4}$.

Making use of Eqs. (6) and (7), in Eq. (4), we obtain

$\frac{\partial T^{\prime}}{\partial t^{\prime}}=\frac{k}{\rho c_{p}} \frac{\partial^{2} T^{\prime}}{\partial y^{2}}+\frac{1}{\rho c_{p}} \frac{16 \sigma^{*} T_{\infty}^{\prime 3}}{3 k^{*}} \frac{\partial^{2} T^{\prime}}{\partial y^{2}}$,

Introducing following non-dimensional variables

$\eta=y / U_{0} t_{0}, u=u^{\prime} / U_{0}, w=w^{\prime} / U_{0}, t=t^{\prime} / t_{0}$ and

$T=\left(T^{\prime}-T_{\infty}^{\prime}\right) /\left(T_{w}^{\prime}-T_{\infty}^{\prime}\right)$,

the Eqs. (2), (3) and (8), in non-dimensional form, become

$$
\begin{aligned}
& \frac{\partial u}{\partial t}+2 K^{2} w=\frac{\partial^{2} u}{\partial \eta^{2}}-M u-\frac{u}{K_{1}}+G_{r} T \\
& \frac{\partial w}{\partial t}-2 K^{2} u=\frac{\partial^{2} w}{\partial \eta^{2}}-M w-\frac{w}{K_{1}}, \\
& \frac{\partial T}{\partial t}=\frac{(1+N)}{P_{r}} \frac{\partial^{2} T}{\partial \eta^{2}}
\end{aligned}
$$


G.S. Seth et al. / JAFM, Vol. 6, No. 1, pp. 27-38, 2013.

where $K^{2}=\Omega v / U_{0}^{2}$ is rotation parameter, $K_{1}=K_{1} U_{0}^{2} / v^{2}$ is porosity parameter, $M=\sigma B_{0}^{2} v / \rho U_{0}^{2}$ is magnetic parameter, $G_{r}=g \beta^{\prime} v\left(T_{w}^{\prime}-T_{\infty}^{\prime}\right) / U_{0}{ }^{3}$ is Grashof number, $P_{r}=\rho v c_{p} / k$ is Prandtl number and $N=16 \sigma^{*} T_{\infty}^{\prime} 3 / 3 k k^{*}$ is radiation parameter.

According to above non-dimensionalization process, the characteristic time $t_{0}$ can be defined as

$t_{0}=v / U_{0}^{2}$.

Making use of (12) the initial and boundary conditions (5), in non-dimensional form, reduce to

$u=w=0, T=0$ for $\eta \geq 0$ and $t \leq 0$,

$u=1, w=0$ at $\eta=0$ for $t>0$,

$T=t$ at $\eta=0$ for $0<t \leq 1$,

$T=1$ at $\eta=0$ for $t>1$,

$u \rightarrow 0, w \rightarrow 0, T \rightarrow 0$ as $\eta \rightarrow \infty$ for $t>0$.

$\tilde{f}=1 / s, \tilde{T}=\left(1-e^{-s}\right) / s^{2}$ at $\eta=0$,

$\tilde{f} \rightarrow 0, \tilde{T} \rightarrow 0$ as $\eta \rightarrow \infty$.

The solution of Eqs. (16) and (17) subject to the boundary conditions (18) are given by

$\tilde{T}(\eta, s)=\frac{1-e^{-s}}{s^{2}} e^{-\eta \sqrt{a s}}$,

$\tilde{f}(\eta, s)=\frac{1}{s} e^{-\eta \sqrt{s+\lambda}}$

$+\alpha \frac{1-e^{-s}}{s^{2}(s-\beta)}\left\{e^{-\eta \sqrt{s+\lambda}}-e^{-\eta \sqrt{a s}}\right\}$

where

$\left.\begin{array}{l}\lambda=\left(M+\frac{1}{K_{1}}-2 i K^{2}\right), \\ \alpha=G_{r} /(a-1), \beta=\lambda /(a-1) .\end{array}\right\}$

Taking inverse Laplace transform of Eqs. (19) and (20), the exact solution for the fluid temperature $T(\eta, t)$ and fluid velocity $f(\eta, t)=u(\eta, t)+i w(\eta, t)$ is obtained and is expressed in the following form

$\frac{\partial f}{\partial t}-2 i K^{2} f=\frac{\partial^{2} f}{\partial \eta^{2}}-M f-\frac{1}{K_{1}} f+G_{r} T$.

The initial and boundary conditions (13), in combined form, become

$f=0, T=0$ for $\eta \geq 0$ and $t \leq 0$,

$f=1$ at $\eta=0$ for $t>0$,

$T=t$ at $\eta=0$ for $0<t \leq 1$

$T=1$ at $\eta=0$ for $t>1$,

$f \rightarrow 0, T \rightarrow 0$ as $\eta \rightarrow \infty$ for $t>0$.

It is evident from the Eqs. (11) and (14) that the energy Eq. (11) is uncoupled from the Eq. (14). Therefore, we can obtain first the solution for the fluid temperature $T(\eta, t)$ by solving Eq. (11) and then using it in Eq. (14) the solution for $f(\eta, t)$ can be obtained.

Applying Laplace transform technique, the Eqs. (11) and (14) with the help of (15a) reduce to

$\frac{d^{2} \tilde{f}}{d \eta^{2}}-\left(s+M+\frac{1}{K_{1}}-2 i K^{2}\right) \tilde{f}+G_{r} \tilde{T}=0$

$\frac{d^{2} \tilde{T}}{d \eta^{2}}-s a \tilde{T}=0$

where $a=P_{r} /(1+N)$ and $\tilde{f}(\eta, s)$ and $\tilde{T}(\eta, s)$ are Laplace transforms of $f(\eta, t)$ and $T(\eta, t)$ defined by $\tilde{f}(\eta, s)=\int_{0}^{\infty} f(\eta, t) e^{-s t} d t \quad$ and $\quad \tilde{T}(\eta, s)=\int_{0}^{\infty} T(\eta, t) e^{-s t} d t$, $T(\eta, t)=P(\eta, t)-H(t-1) P(\eta, t-1)$,

$f(\eta, t)=\frac{1}{2}\left[e^{\eta \sqrt{\lambda}} \operatorname{erfc}\left(\frac{\eta}{2 \sqrt{t}}+\sqrt{\lambda t}\right)\right]$

$+\frac{1}{2}\left[e^{-\eta \sqrt{\lambda}} \operatorname{erfc}\left(\frac{\eta}{2 \sqrt{t}}-\sqrt{\lambda t}\right)\right]$

$+\alpha[F(\eta, t)-H(t-1) F(\eta, t-1)]$,

where

$P(\eta, t)=\left(\frac{a \eta^{2}}{2}+t\right) \operatorname{erfc}\left(\frac{\eta \sqrt{a}}{2 \sqrt{t}}\right)-\sqrt{\frac{a t}{\pi}} \eta e^{-\frac{a \eta^{2}}{4 t}}$,

$F(\eta, t)=\frac{e^{\beta t}}{2 \beta^{2}}\left\{e^{\eta \sqrt{\lambda+\beta}} \operatorname{erfc}\left(\frac{\eta}{2 \sqrt{t}}+\sqrt{(\lambda+\beta) t}\right)\right\}$

$+\frac{e^{\beta t}}{2 \beta^{2}}\left\{e^{-\eta \sqrt{\lambda+\beta}} \operatorname{erfc}\left(\frac{\eta}{2 \sqrt{t}}-\sqrt{(\lambda+\beta) t}\right)\right\}$

$-\left\{\frac{1}{2 \beta^{2}}+\frac{1}{\beta}\left(\frac{t}{2}+\frac{\eta}{4 \sqrt{\lambda}}\right)\right\} e^{\eta \sqrt{\lambda}} \operatorname{erfc}\left(\frac{\eta}{2 \sqrt{t}}+\sqrt{\lambda t}\right)$

$-\left\{\frac{1}{2 \beta^{2}}+\frac{1}{\beta}\left(\frac{t}{2}-\frac{\eta}{4 \sqrt{\lambda}}\right)\right\} \times e^{-\eta \sqrt{\lambda}} \operatorname{erfc}\left(\frac{\eta}{2 \sqrt{t}}-\sqrt{\lambda t}\right)$.

$-\frac{e^{\beta t}}{2 \beta^{2}}\left\{e^{\eta \sqrt{\beta a}} \operatorname{erfc}\left(\frac{\eta \sqrt{a}}{2 \sqrt{t}}+\sqrt{\beta t}\right)+e^{-\eta \sqrt{\beta a}} \times \operatorname{erfc} \frac{\eta \sqrt{a}}{2 \sqrt{t}}-\sqrt{\beta t}\right\}$

$+\left\{\frac{1}{\beta^{2}} \operatorname{erfc}\left(\frac{\eta \sqrt{a}}{2 \sqrt{t}}\right)+\frac{1}{\beta}\left\{\left(\frac{a \eta^{2}}{2}+t\right) \operatorname{erfc}\left(\frac{\eta \sqrt{a}}{2 \sqrt{t}}\right)-\sqrt{\frac{a t}{\pi}} \eta e^{-\frac{a \eta^{2}}{4 t}}\right\}\right.$

In the Eqs. (22) to (25), erfc $(x)$ is the complementary error function and $H(t-1)$ is the Heaviside unit step function.

( $s>0$ being Laplace transform parameter).

The boundary conditions (15b) to (15e) become 


\section{Solution in Case of Isothermal Plate}

The analytical solution for the fluid temperature and velocity, presented by (22) and (23) respectively, is obtained for unsteady hydromagnetic free convection flow of a viscous incompressible electrically conducting fluid near a vertical moving plate with ramped temperature. In order to highlight the effects of ramped temperature distribution within the plate on the fluid flow, it may be worthwhile to compare such a flow with the one near a moving plate with uniform temperature. Taking into account the assumptions made in the present study, the solution for the fluid temperature and velocity for the fluid flow near a vertical moving isothermal plate is obtained and expressed as

$$
\begin{aligned}
& T(\eta, t)=\operatorname{erfc}\left(\frac{\eta}{2} \sqrt{\frac{a}{t}}\right), \\
& f(\eta, t)=\frac{\left(1-\alpha^{\prime}\right)}{2}\left[e^{\eta \sqrt{\lambda}} \operatorname{erfc}\left(\frac{\eta}{2 \sqrt{t}}+\sqrt{\lambda t}\right)+e^{-\eta \sqrt{\lambda}} \operatorname{erfc}\left(\frac{\eta}{2 \sqrt{t}}-\sqrt{\lambda t}\right)\right] \\
& +\frac{\alpha^{\prime} e^{\beta t}}{2}\left[e^{\eta \sqrt{\lambda+\beta}} \operatorname{erfc}\left(\frac{\eta}{2 \sqrt{t}}+\sqrt{(\lambda+\beta) t}\right)\right] \\
& +\frac{\alpha^{\prime} e^{\beta t}}{2}\left[e^{-\sqrt{\lambda+\beta}} \operatorname{erfc}\left(\frac{\eta}{2 \sqrt{t}}-\sqrt{(\lambda+\beta) t}\right)\right] \\
& +\alpha^{\prime} \operatorname{erfc}\left(\frac{\eta}{2} \sqrt{\frac{a}{t}}\right)-\frac{\alpha^{\prime} e^{\beta t}}{2}\left[e^{\eta \sqrt{\beta a}} \text { erfc }\left(\frac{\eta}{2} \sqrt{\frac{a}{t}}+\sqrt{\beta t}\right)\right] \\
& -\frac{\alpha^{\prime} e^{\beta t}}{2}\left[e^{-\eta \sqrt{\beta a}} \operatorname{erfc}\left(\frac{\eta}{2} \sqrt{\frac{a}{t}}-\sqrt{\beta t}\right)\right],
\end{aligned}
$$

where $\alpha^{\prime}=\alpha / \beta$.

\section{NUSSELT NUMBER AND SKIN FRICTION}

The expressions for the Nusselt number and skin friction, which are measures of the rate of heat transfer and shear stress at the plate respectively, are presented in the following form for the moving plate with ramped temperature

$$
\begin{aligned}
& N u=-\left.\frac{\partial T}{\partial \eta}\right|_{\eta=0}=2 \sqrt{\frac{a}{\pi}}[\sqrt{t}-\sqrt{t-1} H(t-1)], \\
& \left(\tau_{x}+i \tau_{z}\right)=\left.\frac{\partial f}{\partial \eta}\right|_{\eta=0}=\sqrt{\lambda}(\operatorname{erfc}(\sqrt{\lambda t})-1)-\frac{1}{\sqrt{\pi t}} e^{-\lambda t} \\
& +\alpha[R(t)-H(t-1) R(t-1)],
\end{aligned}
$$

where

$$
\begin{aligned}
& R(t)=\frac{e^{\beta t}}{\beta^{2}}\left\{\sqrt{(\lambda+\beta)}(\operatorname{erfc}(\sqrt{(\lambda+\beta) t})-1)-\frac{1}{\sqrt{\pi t}} e^{-(\lambda+\beta) t}\right\} \\
& +\frac{1}{2 \beta \sqrt{\lambda}}(1-\operatorname{erfc}(\sqrt{\lambda t})) \\
& +\frac{1}{\beta}\left(t+\frac{1}{\beta}\right)\left\{\sqrt{\lambda}(1-\operatorname{erfc}(\sqrt{\lambda t}))+\frac{1}{\sqrt{\pi t}} e^{-\lambda t}\right\} \\
& -\frac{e^{\beta t}}{\beta^{2}}\left\{\sqrt{\beta a}(\operatorname{erfc}(\sqrt{\beta t})-1)-\sqrt{\frac{a}{\pi t}} e^{-\beta t}\right\} \\
& -\frac{1}{\beta} \sqrt{\frac{a}{\pi}}\left(2 \sqrt{t}+\frac{1}{\beta \sqrt{t}}\right) .
\end{aligned}
$$

The expressions for the Nusselt number and skin friction for isothermal moving plate are given by

$$
\begin{aligned}
& N u=\sqrt{a / \pi t}, \\
& \left(\tau_{x}+i \tau_{z}\right)=\left(1-\alpha^{\prime}\right)\left\{\sqrt{\lambda}(\operatorname{erfc}(\sqrt{\lambda t})-1)-\frac{1}{\sqrt{\pi t}} e^{-\lambda t}\right\} \\
& +\alpha^{\prime} e^{\beta t}\left\{\sqrt{(\lambda+\beta)}(\operatorname{erfc}(\sqrt{(\lambda+\beta) t})-1)-\frac{1}{\sqrt{\pi t}} e^{-(\lambda+\beta) t}\right\} \\
& -\alpha^{\prime} e^{\beta t}\left\{\sqrt{\beta a}(\operatorname{erfc}(\sqrt{\beta t})-1)-\sqrt{\frac{a}{\pi t}} e^{-\beta t}\right\}-\alpha^{\prime} \sqrt{\frac{a}{\pi t}}
\end{aligned}
$$

It is evident from the expressions (28) and (31) that, for a given time $t$, Nusselt number $N u$, in both the cases, is proportional to $\sqrt{P_{r} /(N+1)}$ i.e. Nusselt number $N u$ increases with the increase in Prandtl number $P_{r}$ whereas it decreases with the increase in radiation parameter $N$. This implies that radiation reduces rate of heat transfer at the plate. Also Nusselt number $N u$ for liquid metal $\left(\mathrm{P}_{\mathrm{r}}=0.01\right)$ is smaller than that of ionized air $\left(\mathrm{P}_{\mathrm{r}}=0.71\right)$ and water $\left(\mathrm{P}_{\mathrm{r}}=7\right)$. It is interesting to note from (28) and (31) that an increase in time $t$ leads to an increase in the Nusselt number for ramped temperature plate whereas it leads to a decrease in the Nusselt number for isothermal plate.

\section{RESULTS AND DISCUSSION}

To study the effects of magnetic field, rotation, free convection, radiation, porosity of medium and time on the flow-field the numerical values of fluid velocity are displayed graphically versus boundary layer coordinate $\eta$ in Figs. 1 to 12 for various values of magnetic parameter $\mathrm{M}$, rotation parameter $K^{2}$, Grashof number $G_{r}$, radiation parameter $N$, porosity parameter $K_{1}$ and time $t$ taking $P_{r}=0.71$ (ionized air). It is noticed from Figs. 1 and 2 that an increase in the magnetic parameter $M$ leads to a decrease in the primary velocity $u$ and the secondary velocity $w$ for both ramped temperature and isothermal plates. This is due to the fact that the application of a transverse magnetic field to an electrically conducting fluid gives rise to a resistive force which is known as Lorentz force. This force has the tendency to retard the fluid motion in the boundary layer region. It is evident from Figs. 3 and 4 that, for both ramped temperature and isothermal plates, an increase in rotation parameter $K^{2}$ leads to a decrease in the primary velocity $u$ and an increase in the secondary velocity $w$ in the region near the plate. The characteristics of secondary velocity $w$, in the region away from the plate, is different than that in the region near the plate. This implies that rotation retards fluid flow in the primary flow direction and accelerates fluid flow in the secondary flow direction in the boundary layer region. This may be attributed to the fact that when the frictional layer at the moving plate is suddenly set into the motion then the Coriolis force acts as a constraint in the main fluid flow i.e. in the fluid flow in the primary flow direction to generate cross flow i.e. secondary flow. It is found from Figs. 5 to 12 that both the primary and secondary velocities increase on increasing Grashof number $G_{r}$, radiation parameter $N$, porosity parameter $K_{1}$ and time $t$ for both ramped temperature and isothermal plates. This implies that free 
convection, radiation, porosity of medium and time exert accelerating influence on the primary flow as well as on the secondary flow. It is also evident from Figs. 1 to 12 that the

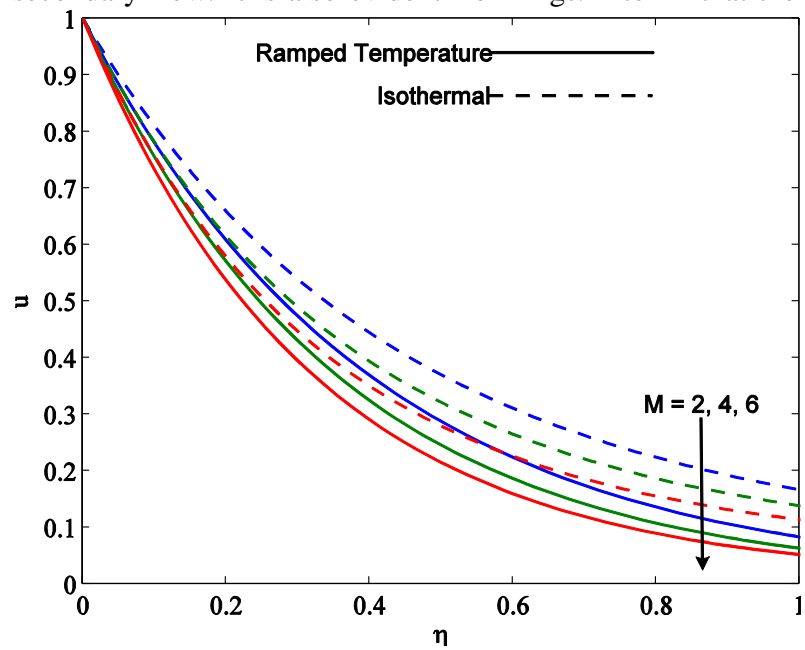

Fig. 1. Profiles of primary velocity when $K^{2}=2, G_{r}=2, N=1$, $\mathrm{K}_{1}=0.2$ and $\mathrm{t}=0.5$

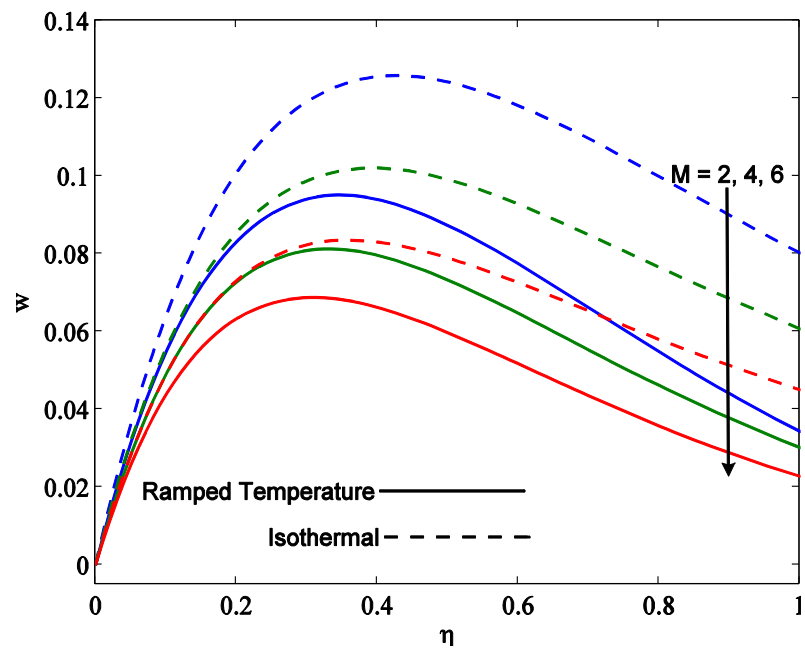

Fig. 2. Profiles of secondary velocity when $K^{2}=2, G_{r}=2, N=1$, $\mathrm{K}_{1}=0.2$ and $\mathrm{t}=0.5$

In order to have physical view of fluid temperature the profiles of fluid temperature are drawn versus boundary layer coordinate $\eta$ in Figs. 13 to 15 for various values of radiation parameter $N$, Prandtl number $P_{r}$ and time $t$. It is evident from Figs. 13 to 15 that the fluid temperature $T$ increases on increasing radiation parameter $N$ or time $t$ and it decreases on increasing Prandtl number $P_{r}$ for both ramped temperature and isothermal plates. This implies that radiation tends to increase fluid temperature and fluid temperature increases with the passage of time $t$ in both the cases. Prandtl number $P_{r}$, which is a measure of relative importance of viscosity and thermal conductivity of the fluid, has tendency to reduce fluid temperature in both the cases. Also for liquid metals (i.e. for $P_{r}=0.01$ and 0.03 ) the variation in fluid fluid velocities are faster in the case of isothermal plate than that in the case of ramped temperature plate.

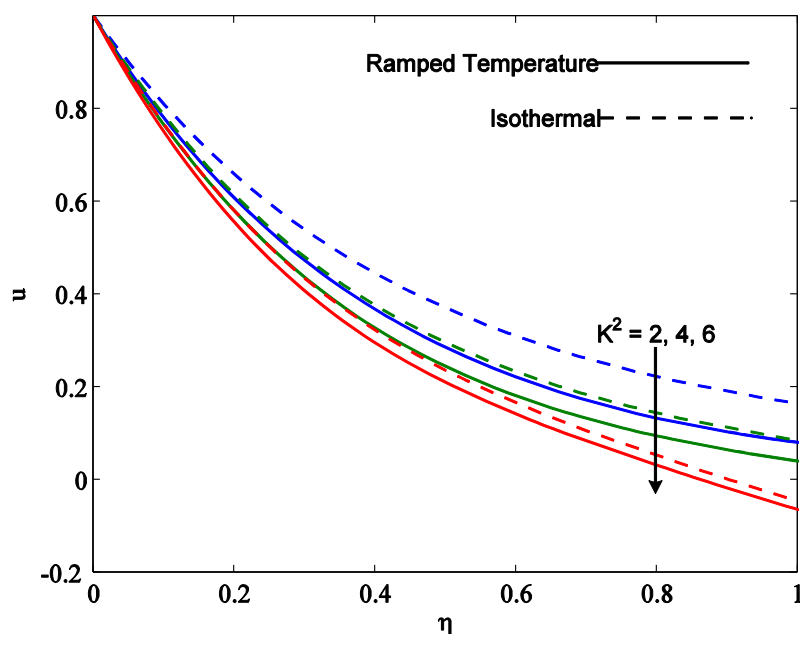

Fig. 3. Profiles of primary velocity when $M=2, G_{r}=2, N=1$, $\mathrm{K}_{1}=0.2$ and $\mathrm{t}=0.5$

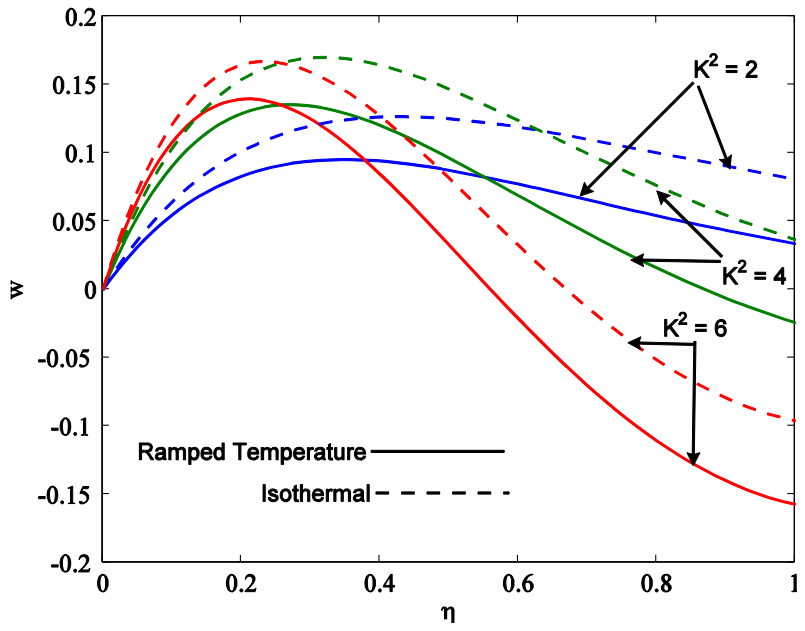

Fig. 4. Profiles of secondary velocity when $M=2, G_{r}=2, N=1$, $\mathrm{K}_{1}=0.2$ and $\mathrm{t}=0.5$

temperature is very slow on increasing boundary layer coordinate $\eta$ whereas for ionized air $\left(P_{r}=0.5\right.$ and 0.71$)$ the change in fluid temperature is very rapid with respect to $\eta$. Fluid temperature $T$ is lower in the case of ramped temperature plate than that in the case of isothermal plate.

The numerical values of the primary and secondary skin frictions $\tau_{x}$ and $\tau_{z}$ for ramped temperature plate as well as isothermal plate are displayed in tables 1 to 6 for various values of $M, K^{2}, G_{r}, K_{1}, N$ and $t$ taking $P_{r}=0.71$ whereas that of Nusselt number $N u$ for both ramped temperature and isothermal plates are provided in tabular form in tables 7 and 8 for different values of $N, t$ and $P_{r}$. 
G.S. Seth et al. / JAFM, Vol. 6, No. 1, pp. 27-38, 2013.

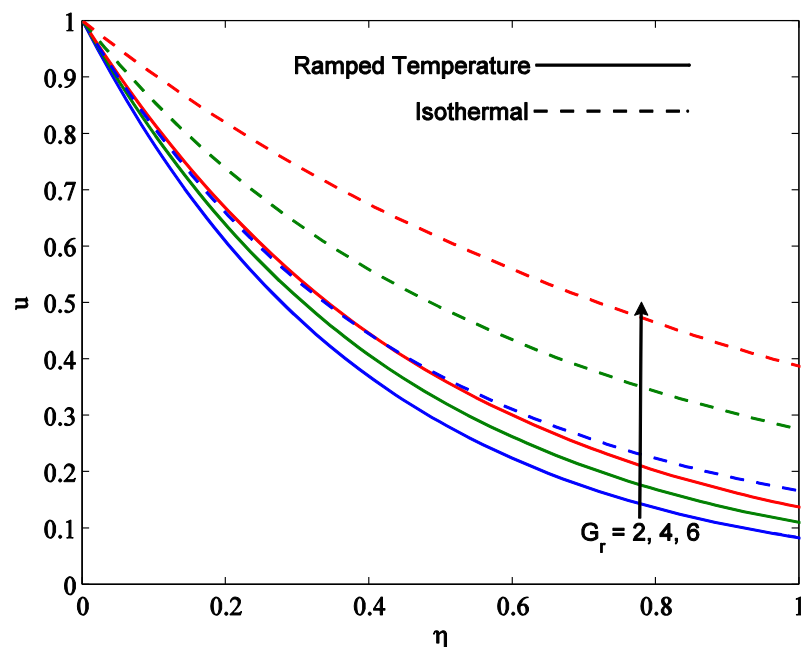

Fig. 5. Profiles of primary velocity when $M=2, K^{2}=2, N=1$, $\mathrm{K}_{1}=0.2$ and $\mathrm{t}=0.5$

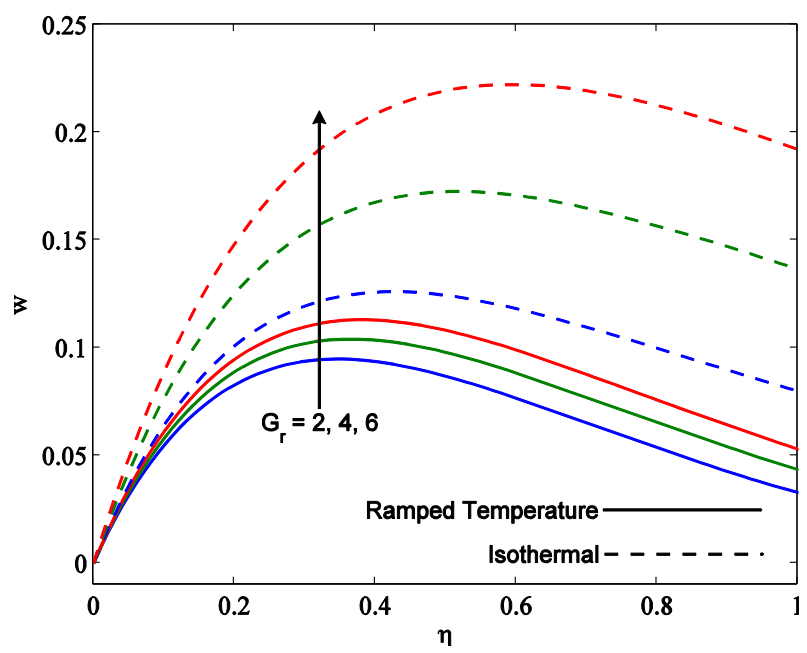

Fig. 6. Profiles of secondary velocity when $M=2, K^{2}=2, N=1$, $\mathrm{K}_{1}=0.2$ and $\mathrm{t}=0.5$

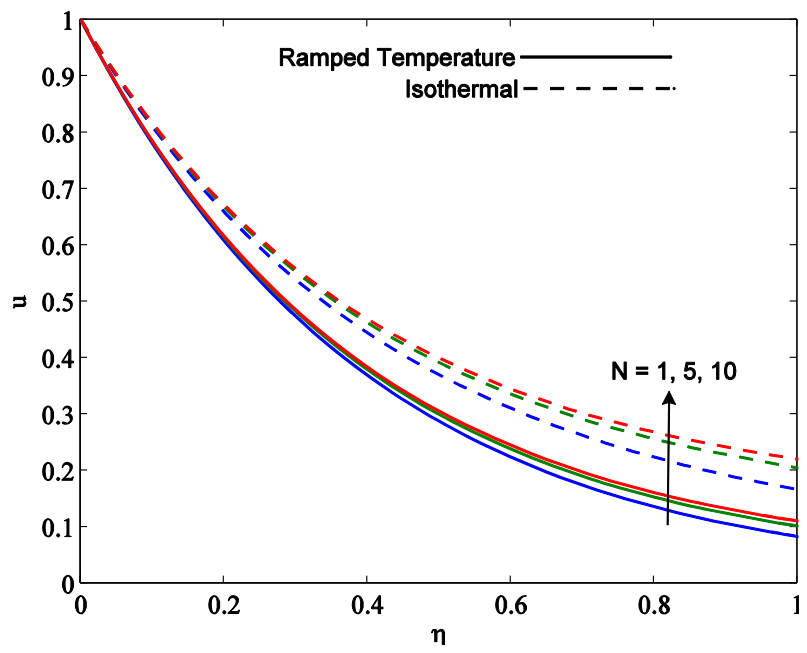

Fig. 7. Profiles of primary velocity when $M=2, K^{2}=2, G_{r}=2$, $\mathrm{K}_{1}=0.2$ and $\mathrm{t}=0.5$

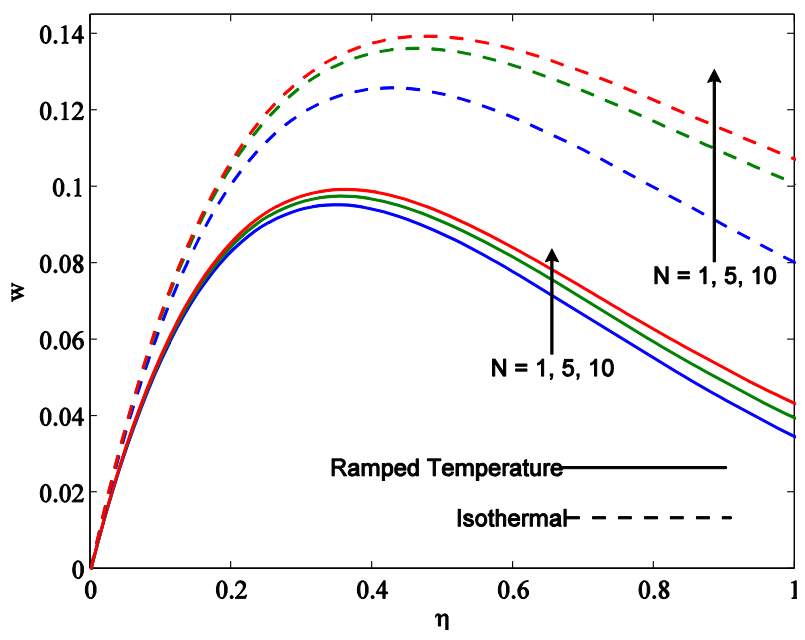

Fig. 8. Profiles of secondary velocity when $M=2, K^{2}=2, G_{r}=2$, $\mathrm{K}_{1}=0.2$ and $\mathrm{t}=0.5$

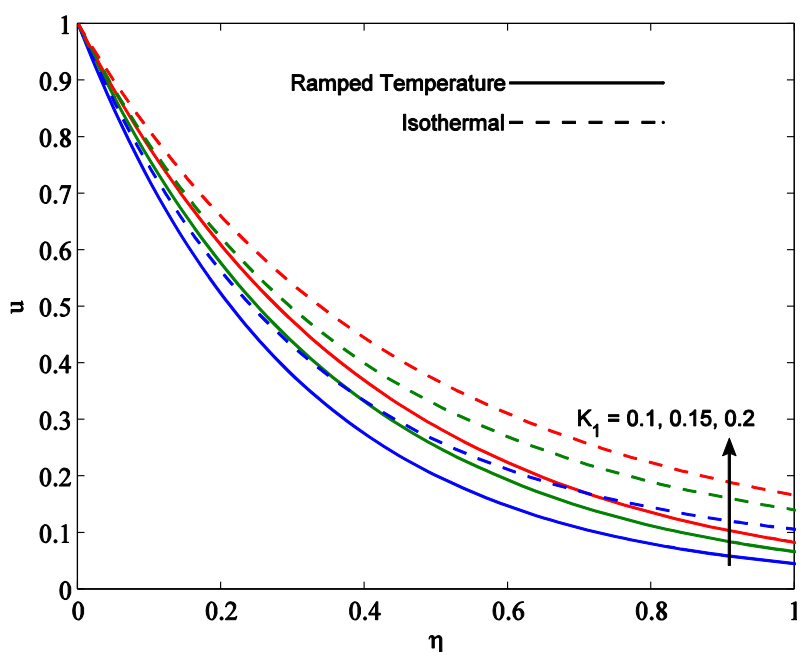

Fig. 9. Profiles of primary velocity when $M=2, K^{2}=2, G_{r}=2$, $\mathrm{N}=1$ and $\mathrm{t}=0.5$

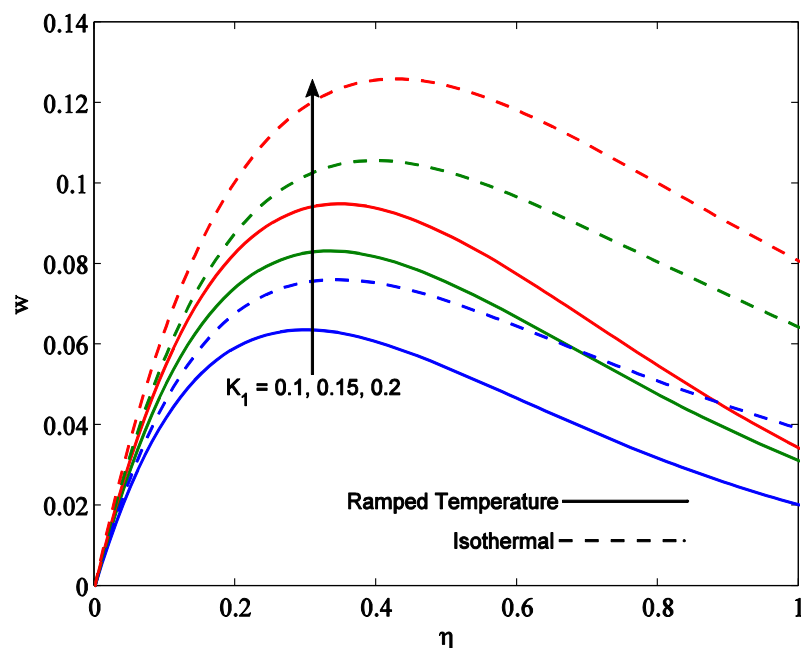

Fig. 10. Profiles of secondary velocity when $M=2, K^{2}=2$, $\mathrm{G}_{\mathrm{r}}=2, \mathrm{~N}=1$ and $\mathrm{t}=0.5$ 


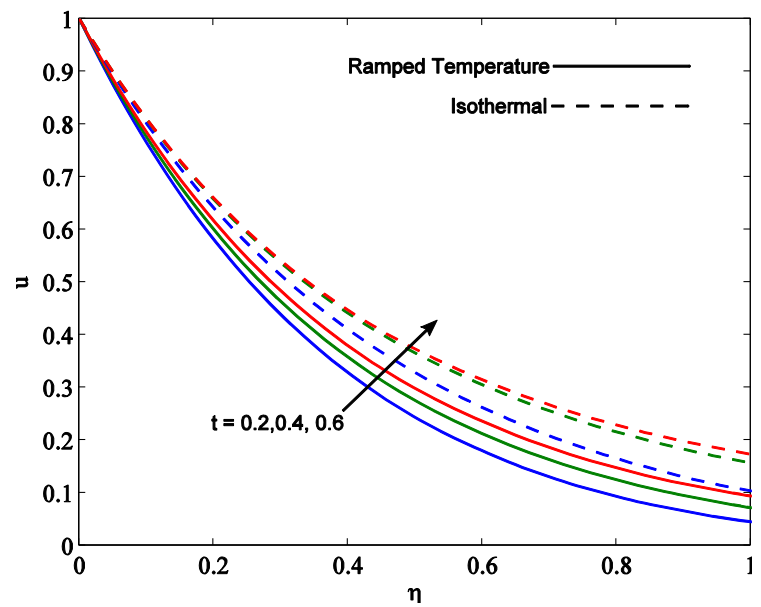

Fig. 11.Profiles of primary velocity when $M=2, K^{2}=2, G_{r}=2$, $\mathrm{N}=1$ and $\mathrm{K}_{1}=0.2$

It is noticed from tables 1 and 2 that, for both ramped temperature and isothermal plates, the primary skin friction i.e. $\tau_{x}$ and secondary skin friction i.e. $\tau_{z}$ increase on increasing either $M$ or $K^{2}$. It is found from tables 3 and 4 that an increase in either $G_{r}$ or $K_{1}$ leads to a decrease in $\tau_{x}$ whereas it leads to an increase in $\tau_{z}$ for both ramped temperature and isothermal plates. It is evident from tables 5 and 6 that, for ramped temperature plate, $\tau_{x}$ decreases on increasing $N$ whereas it increases on increasing time $t$. However, $\tau_{z}$ increases as either $N$ or $t$ increases. For isothermal plate, with an increase in $N, \tau_{x}$ increases when $t=0.2$ and decreases when $t=0.4$ and 0.6. Also $\tau_{x}$ increases on increasing time $t . \tau_{z}$ decreases on increasing $N$ whereas it increases as time $t$ increases. Keeping in view the trend mentioned above of $\tau_{x}$ and $\tau_{z}$ with respect to different parameters we conclude that magnetic field and rotation tend to increase primary as well as secondary skin friction for both ramped temperature and isothermal plates. For both ramped temperature and isothermal plate free convection and porosity of the medium have tendency to reduce primary skin friction whereas it tend to increase

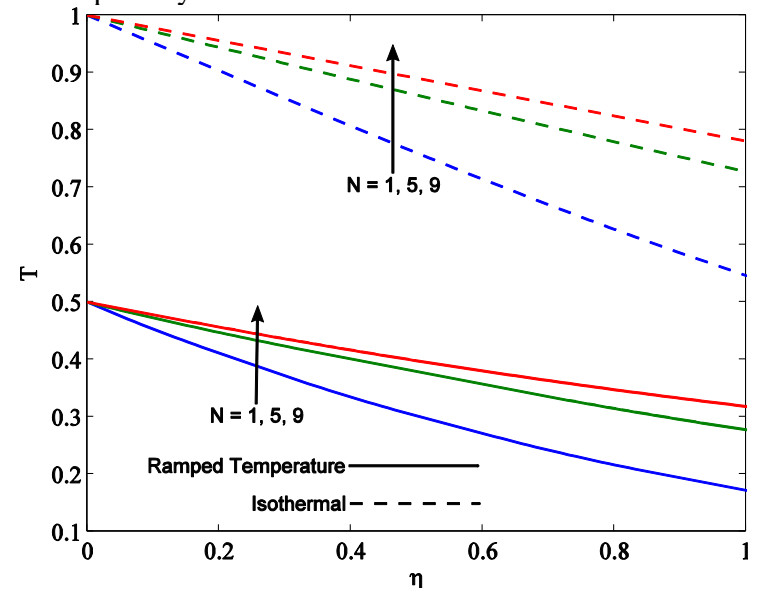

Fig. 13. Profiles of temperature when $P_{r}=0.71$ and $t=0.5$

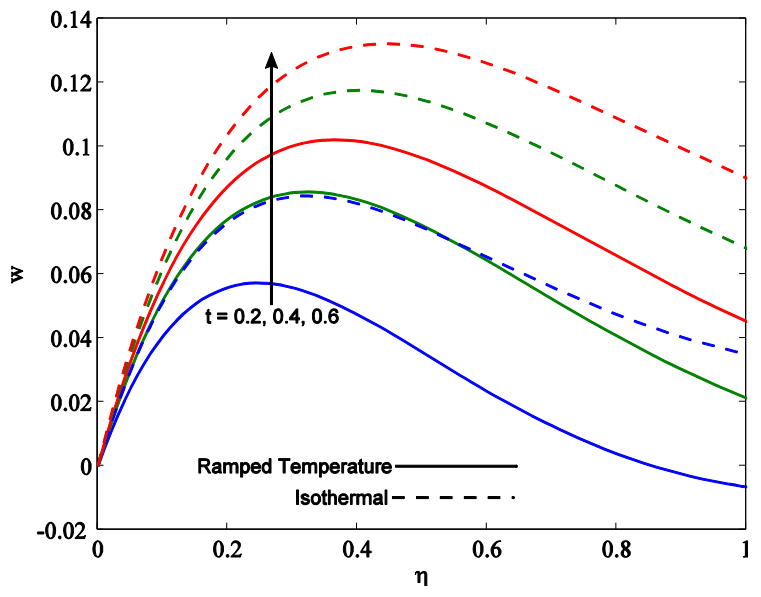

Fig. 12. Profiles of secondary velocity when $M=2, K^{2}=2$, $\mathrm{G}_{\mathrm{r}}=2, \mathrm{~N}=1$ and $\mathrm{K}_{1}=0.2$

secondary skin friction. For ramped temperature plate radiation reduces primary skin friction whereas it tends to increase secondary skin friction. For isothermal plate radiation reduces secondary skin friction. It is also noticed from table 6 that, for isothermal plate, variation in $\tau_{x}$ and $\tau_{z}$ is almost uniform with respect to radiation parameter $N$ when $t$ is large. This implies that the effects of radiation on primary and secondary skin frictions become insignificant with passage of time for isothermal plate. It is evident from tables 7 and 8 that, for both ramped temperature and isothermal plates, Nusselt number $N u$, which is a measure of rate of heat transfer at the plate, decreases on increasing $N$ whereas it increases with the increase in Prandtl number $P_{r}$. For ramped temperature, $\mathrm{Nu}$ increases with the increase in time $t$ whereas it decreases on increasing $t$ for isothermal plate. This implies that radiation reduces rate of heat transfer at the plate for both ramped temperature and isothermal plates. Prandtl number tends to increase rate of heat transfer at the plate for both ramped temperature and isothermal plates. With the increase in time the rate of heat transfer at the plate is reduced for isothermal plate while it is increased for ramped temperature plate.

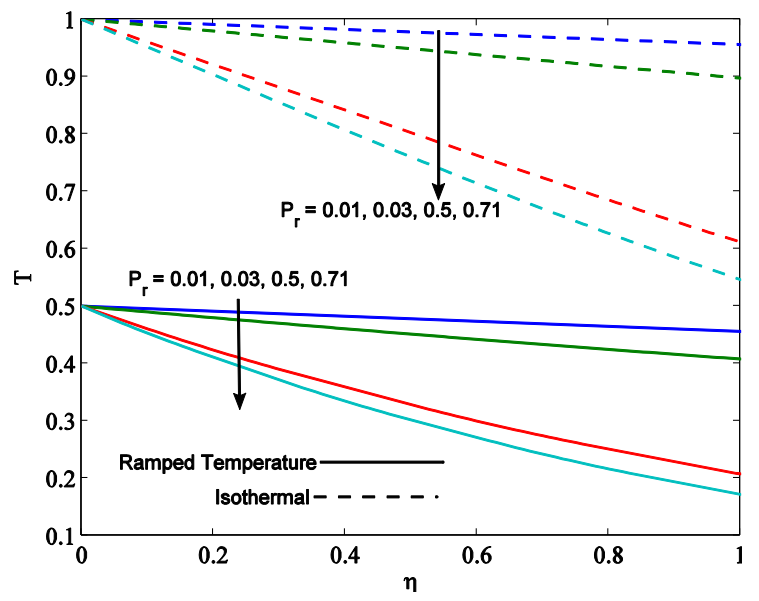

Fig. 14. Profiles of temperature when $\mathrm{N}=1$ and $\mathrm{t}=0.5$ 
G.S. Seth et al. / JAFM, Vol. 6, No. 1, pp. 27-38, 2013.

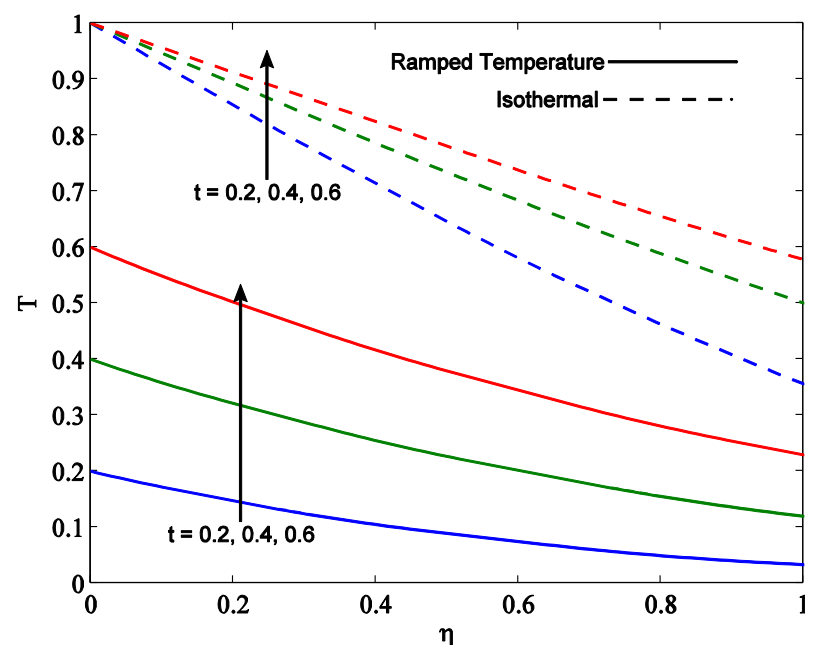

Fig. 15. Profiles of temperature when $P_{r}=0.71$ and $N=1$

Table 1 Skin frictions $\boldsymbol{\tau}_{\mathrm{x}}$ and $\boldsymbol{\tau}_{\mathrm{z}}$ for ramped temperature plate when $\mathrm{G}_{\mathrm{r}}=2, \mathrm{~N}=1, \mathrm{~K}_{1}=0.2$ and $\mathrm{t}=0.5$.

\begin{tabular}{|c|c|c|c|c|c|c|}
\hline \multirow{2}{*}{$\mathrm{M} \downarrow / \mathrm{K}^{2} \rightarrow$} & \multicolumn{3}{|c|}{$-\tau_{x}$} & \multicolumn{3}{c|}{$\tau_{z}$} \\
\cline { 2 - 7 } & 2 & 4 & 6 & 2 & 4 & 6 \\
\hline 2 & 2.4527 & 2.6436 & 2.7299 & 1.2609 & 2.2537 & 2.5595 \\
\hline 4 & 2.8985 & 3.2743 & 3.4381 & 1.2240 & 2.6090 & 3.1152 \\
\hline 6 & 3.2488 & 3.7956 & 4.0836 & 1.1589 & 2.8426 & 3.5936 \\
\hline
\end{tabular}

Table 2 Skin frictions $\boldsymbol{\tau}_{\mathrm{x}}$ and $\boldsymbol{\tau}_{\mathrm{z}}$ for isothermal plate when $\mathrm{G}_{\mathrm{r}}=2, \mathrm{~N}=1, \mathrm{~K}_{1}=0.2$ and $\mathrm{t}=0.5$.

\begin{tabular}{|c|c|c|c|c|c|c|}
\hline \multirow{2}{*}{$\mathrm{M} \downarrow / \mathrm{K}^{2} \rightarrow$} & \multicolumn{3}{|c|}{$-\tau_{x}$} & \multicolumn{3}{|c|}{$\tau_{z}$} \\
\cline { 2 - 7 } & 2 & 4 & 6 & 2 & 4 & 6 \\
\hline 2 & 2.1419 & 2.5541 & 2.6908 & 1.4511 & 2.3511 & 2.6076 \\
\hline 4 & 2.5790 & 3.1654 & 3.3887 & 1.3809 & 2.7148 & 3.1709 \\
\hline 6 & 2.9357 & 3.6714 & 4.0250 & 1.2872 & 2.9528 & 3.6556 \\
\hline
\end{tabular}

Table 3 Skin frictions $\boldsymbol{\tau}_{\mathrm{x}}$ and $\boldsymbol{\tau}_{\mathrm{z}}$ for ramped temperature plate when $\mathrm{M}=2, \mathrm{~N}=1, \mathrm{~K}^{2}=2$ and $\mathrm{t}=0.5$.

\begin{tabular}{|c|c|c|c|c|c|c|}
\hline \multirow{2}{*}{$\mathrm{G}_{\mathrm{r} \downarrow} \downarrow / \mathrm{K}_{1} \rightarrow$} & \multicolumn{3}{|c|}{$-\tau_{x}$} & \multicolumn{3}{c|}{$\tau_{z}$} \\
\hline & 0.1 & 0.15 & 0.2 & 0.1 & 0.15 & 0.2 \\
\hline 2 & 3.4015 & 2.8322 & 2.4527 & 1.1243 & 1.2331 & 1.2609 \\
\hline 4 & 3.2105 & 2.6371 & 2.2634 & 1.1590 & 1.2720 & 1.2977 \\
\hline 6 & 3.0195 & 2.4420 & 2.0741 & 1.1937 & 1.3109 & 1.3344 \\
\hline
\end{tabular}

Table 4 Skin frictions $\boldsymbol{\tau}_{\mathrm{x}}$ and $\boldsymbol{\tau}_{\mathrm{z}}$ for isothermal plate when $\mathrm{M}=2, \mathrm{~N}=1, \mathrm{~K}^{2}=2$ and $\mathrm{t}=0.5$.

\begin{tabular}{|c|c|c|c|c|c|c|}
\hline \multirow{2}{*}{$\mathrm{G}_{\mathrm{r}} \downarrow / \mathrm{K}_{1} \rightarrow$} & \multicolumn{3}{|c|}{$-\tau_{x}$} & \multicolumn{3}{c|}{$\tau_{z}$} \\
\cline { 2 - 7 } & 0.1 & 0.15 & 0.2 & 0.1 & 0.15 & 0.2 \\
\hline 2 & 3.0941 & 2.5127 & 2.1419 & 1.2405 & 1.3954 & 1.4511 \\
\hline 4 & 2.5957 & 1.9982 & 1.6419 & 1.3914 & 1.5965 & 1.6780 \\
\hline 6 & 2.0974 & 1.4838 & 1.1419 & 1.5423 & 1.7977 & 1.9049 \\
\hline
\end{tabular}

Table 5 Skin frictions $\boldsymbol{\tau}_{\mathrm{x}}$ and $\boldsymbol{\tau}_{\mathrm{z}}$ for ramped temperature plate when $\mathrm{M}=2, \mathrm{G}_{\mathrm{r}}=2, \mathrm{~K}^{2}=2$ and $\mathrm{K}_{1}=0.2$.

\begin{tabular}{|c|c|c|c|c|c|c|}
\hline \multirow{2}{*}{$\mathrm{t} \downarrow / \mathrm{N} \rightarrow$} & \multicolumn{3}{|c|}{$-\tau_{x}$} & \multicolumn{3}{c|}{$\tau_{z}$} \\
\cline { 2 - 7 } & 1 & 5 & 10 & 1 & 5 & 10 \\
\hline 0.2 & 2.0247 & 2.0115 & 2.0064 & 0.6200 & 0.6300 & 0.6346 \\
\hline 0.4 & 2.3453 & 2.3266 & 2.3195 & 1.1687 & 1.1830 & 1.1896 \\
\hline 0.6 & 2.5013 & 2.4777 & 2.4688 & 1.3149 & 1.3340 & 1.3424 \\
\hline
\end{tabular}


G.S. Seth et al. / JAFM, Vol. 6, No. 1, pp. 27-38, 2013.

Table 6 Skin frictions $\boldsymbol{\tau}_{\mathrm{x}}$ and $\boldsymbol{\tau}_{\mathrm{z}}$ for isothermal plate when $\mathrm{M}=2, \mathrm{G}_{\mathrm{r}}=2, \mathrm{~K}^{2}=2$ and $\mathrm{K}_{1}=0.2$.

\begin{tabular}{|c|c|c|c|c|c|c|}
\hline \multirow{2}{*}{$\mathrm{t} \downarrow / \mathrm{N} \rightarrow$} & \multicolumn{3}{|c|}{$-\tau_{x}$} & \multicolumn{3}{c|}{$\tau_{z}$} \\
\cline { 2 - 7 } & 1 & 5 & 10 & 1 & 5 & 10 \\
\hline 0.2 & 1.6666 & 1.6774 & 1.6803 & 0.7994 & 0.7925 & 0.7924 \\
\hline 0.4 & 2.0021 & 2.0010 & 2.0005 & 1.3688 & 1.3678 & 1.3668 \\
\hline 0.6 & 2.2290 & 2.2290 & 2.2288 & 1.4905 & 1.4907 & 1.4908 \\
\hline
\end{tabular}

Table 7 Nusselt number Nu when $\mathrm{P}_{\mathrm{r}}=0.71$

\begin{tabular}{|c|c|c|c|c|c|c|}
\hline \multirow{2}{*}{$\mathrm{N} \downarrow / \mathrm{t} \rightarrow$} & \multicolumn{2}{|c|}{ Ramped Temperature Plate } & \multicolumn{3}{|c|}{ Isothermal Plate } \\
\cline { 2 - 7 } & 0.2 & 0.4 & 0.6 & 0.2 & 0.4 & 0.6 \\
\hline 1 & 0.3007 & 0.4252 & 0.5208 & 0.7517 & 0.5315 & 0.4340 \\
\hline 5 & 0.1736 & 0.2455 & 0.3007 & 0.4340 & 0.3069 & 0.2506 \\
\hline 10 & 0.1282 & 0.1813 & 0.2221 & 0.3205 & 0.2266 & 0.1850 \\
\hline
\end{tabular}

Table 8 Nusselt number $\mathrm{Nu}$ when $\mathrm{N}=1$

\begin{tabular}{|c|c|c|c|c|c|c|}
\hline \multirow{2}{*}{$\mathrm{P}_{\mathrm{r}} \downarrow / \mathrm{t} \rightarrow$} & \multicolumn{3}{|c|}{ Ramped Temperature Plate } & \multicolumn{3}{|c|}{ Isothermal Plate } \\
\cline { 2 - 7 } & 0.2 & 0.4 & 0.6 & 0.2 & 0.4 & 0.6 \\
\hline 0.01 & 0.0357 & 0.0505 & 0.0618 & 0.0892 & 0.0631 & 0.0515 \\
\hline 0.03 & 0.0618 & 0.0874 & 0.1070 & 0.1545 & 0.1093 & 0.0892 \\
\hline 0.5 & 0.2523 & 0.3568 & 0.4370 & 0.6308 & 0.4460 & 0.3642 \\
\hline 0.71 & 0.3007 & 0.4252 & 0.5208 & 0.7517 & 0.5315 & 0.4340 \\
\hline
\end{tabular}

\section{CONCLUSION}

This study presents a theoretical investigation of unsteady hydromagnetic free convection boundary layer flow of a viscous incompressible electrically conducting fluid past a ramped temperature impulsively moving plate in a rotating porous medium in the presence of transverse magnetic field and thermal radiation. The significant findings are summarized as:

a). For both ramped temperature and isothermal plates:

(i). Magnetic field tends to retard fluid flow in both the primary and secondary flow directions.

(ii). rotation retards fluid flow in the primary flow direction whereas it accelerates fluid flow in the secondary flow direction in the boundary layer region.

(iii). free convection, radiation, porosity of medium and time exert accelerating influence on the fluid flow in both the primary and secondary flow directions.

(iv). radiation tends to increase fluid temperature whereas with passage of time fluid temperature increases.

(v). Prandtl number has tendency to reduce fluid temperature.

b) (i). For both ramped temperature and isothermal plates magnetic field and rotation tend to increase primary as well secondary skin friction whereas free convection and porosity of the medium have tendency to reduce primary skin friction and it tend to increase secondary skin friction.

(ii). For ramped temperature plate radiation reduces primary skin friction whereas it tends to increase secondary skin friction. For isothermal plate radiation has tendency to reduce secondary skin friction.

c). For both ramped temperature and isothermal plates radiation tends to reduce rate of heat transfer at the plate whereas Prandtl number has tendency to increase rate of heat transfer at the plate. With the increase in time the rate of heat transfer at the plate is reduced for isothermal plate while it is increased for ramped temperature plate.

\section{REFERENCES}

Aldoss, T.K., M.A. Al-Nimr, M.A. Jarrah and B.J. Al-Shaer (1995). Magnetohydrodynamic mixed convection from a vertical plate embedded in a porous medium. Numer. Heat Transfer 28, 635-645.

Azzam, G.E.A. (2002). Radiation effects on the MHD mixed free-forced convective flow past a semi-infinite moving vertical plate for high temperature differences. Phys. Scripta, 66, 71-76.

Bakier, A.Y. and Gorla, R.S.R. (1996). Thermal Radiation Effect on Mixed Convection from Horizontal Surfaces in Saturated Porous Media. Transport in Porous media 23, 357-363

Beg, O.A., Takhar, H.S., Kumari, M. and Nath, G. (2001). Computational fluid dynamics modelling of buoyancy induced viscoelastic flow in a porous medium with magnetic field effects. Int. J. Appl. Mech. Eng. 6, 187210 .

Bestman, A.R. and Adjepong, S.K. (1998). Unsteady hydromagnetic free-convection flow with radiative heat transfer in a rotating fluid. Astrophys. Space Sci. 143, 7380.

Brewster, M.Q. (1992). Thermal Radiative Transfer and Properties, John Wiley \& Sons, New York. 
Kao, T.T. (1975). Laminar free convective heat transfer response along a vertical flat plate with step jump in surface temperature. Lett. Heat Mass Transfer 2, 419-428.

Chamkha, A.J. (2000). Thermal radiation and buoyancy effects on hydromagnetic flow over an accelerating permeable surface with heat source or sink. Int. J. Engng. Sci. 38, 1699-1712.

Chandran, P., Sacheti, N.C. and Singh, A.K. (2005). Natural Convection near a vertical plate with ramped wall temperature. Heat Mass Transfer 41, 459-464.

Cheng, P. and Minkowycz, W.J. (1977). Free convection about a vertical plate embedded in a porous medium with application to heat transfer form a dike. J. Geophys. Res. $82,2040-2044$.

Cramer, K.R. and Pai, S.I. (1973). Magnetofluiddynamics for Engineers and Applied physicists. McGraw Hill Book Company, New York.

Hayday, A.A., Bowlus, D.A. and McGraw, R.A. (1967). Free convection from a vertical plate with step discontinuities in surface temperature. ASME J. Heat Transfer 89, 244250 .

Helmy, K.A. (1998). MHD unsteady free convection flow past a vertical porous plate. ZAMM 78, 255-270.

Hossain, M.A. and Takhar, H.S. (1996). Radiation effect on mixed convection along a vertical plate with uniform surface temperature, Heat Mass Transfer 31, 243-248.

Hsieh, J.C., Chen, T.S. and Armaly, B.F. (1993). Nonsimilarity solutions for mixed convection from vertical surfaces in porous media, variable surface temperature or heat flux. Int. J. Heat Mass Transfer 36, 1485-1493.

Ibrahim, F.S, Hassanien, I.A. and Bakr, A.A. (2004). Unsteady Magnetohydrodynamic micropolar fluid flow and heat transfer over a vertical porous medium in the presence of thermal and mass diffusion with constant heat source. Canad. J. Phys. 82, 775-790.

Israel-Cookey, C., Ogulu, A. and Omubo-Pepple, V.B. (2003). Influence of viscous dissipation and radiation on unsteady MHD free-convection flow past an infinite heated vertical plate in a porous medium with timedependent suction. Int. J. Heat Mass Transfer 46, 23052311.

Jha, B.K. (1991). MHD free-convection and mass-transform flow through a porous medium. Astrophys. Space Sci. 175, 283-289.

Kelleher, M. (1971). Free convection from a vertical plate with discontinuous wall temperature. ASME J. Heat Transfer 93, 349-356.
Kim, Y.J. (2000). Unsteady MHD convective heat transfer past a semi-infinite vertical porous moving plate with variable suction. Int. J. Engng. Sci. 38, 833-845.

Kim, Y.J. and Fedorov, A.G. (2003). Transient mixed radiative convection flow of a micropolar fluid past a moving, semi-infinite vertical porous plate. Int. J. Heat Mass Trasnfer 46, 1751-1758.

Lai, F.C. and Kulacki, F.A. (1991). Non-Darcy mixed convection along a vertical wall in a saturated porous medium. J. Heat Mass Transfer 113, 252-254.

Lee, S. and Yovanovich, M.M. (1991). Laminar natural convection from a vertical plate with a step change in wall temperature. ASME J. Heat Transfer 113, 501-504.

Mahmoud Mostafa, A.A. (2009). Thermal radiation effect on unsteady MHD free convection flow past a vertical plate with temperature dependent viscosity. The Canad. J. Chem. Eng. 87, 47-52.

Mbeledogu, I.U. and Ogulu, A. (2007). Heat and mass transfer of an unsteady MHD natural convection flow of a rotating fluid past a vertical porous flat plate in the presence of radiative heat transfer. Int. J. Heat Mass Transfer 50, 1902-1908.

Meyer, R.C. (1958). On reducing aerodynamic heat-transfer rates by magnetohydrodynamic techniques. J. Aero. Sci. $25,561-572$.

Minkowycz, W.J., Cheng, P. and Chang, C.H. (1985). Mixed convection about a non-isothermal cylinder and sphere in a porous medium. Numer. Heat Transfer 8, 349-359.

Nakayama, A. and Koyama, H. (1987). A general similarity transformation for combined free and forced convection flows within a fluid saturated porous medium. ASME J. Heat Transfer 109, 1041-1045.

Nield, D.A. and Bejan, A. (2006). Convection in porous media, third edition, Springer Science, USA.

Pop, I. and Ingham, D.B. (eds.) (2002). Transport Phenomena in Porous Media-II, Pergamon Press, UK.

Prasad, V.R., Bhaskar Reddy, N. and Muthucumaraswamy, R.(2007). Radiation and mass transfer effects on twodimensional flow past an impulsively started infinite vertical plate. Int. J. Thermal Sci. 46, 1251-1258.

Ranganathan, P. and R. Viskanta (1984). Mixed convection boundary layer flow along a vertical surface in a porous medium. Numer. Heat Transfer 7, 305-317.

Raptis, A. and Kafousias, N.(1982). Heat transfer in flow through a porous medium bounded by an infinite vertical plate under the action of a magnetic field. Int. J. Energy Res. 6, 241-245. 
G.S. Seth et al. / JAFM, Vol. 6, No. 1, pp. 27-38, 2013.

Raptis, A. (1986). Flow through a porous medium in the presence of a magnetic field. Int. J. Energy Res. 10, 97100.

Sahoo, D.G. and Sahoo, P.K. (1986). Hydromagnetic free convection and mass transfer through a porous medium. Ind. J. Tech. 24, 553-556.

Takhar, H.S. and Ram, P.C. (1991). Free convection in hydromagnetic flows of a viscous heat-generating fluid with wall temperature oscillation and Hall currents. Astrophys. Space Sci. 183, 193-198.
Takhar, H.S. and Ram, P.C. (1994). Magnetohydrodynamic free convection flow of water at $4^{0} \mathrm{C}$ through a porous medium. Int. Comm. Heat Mass Transfer 21, 371-376.

Takhar, H.S., Gorla, R.S.R. and Soundalgekar, V.M. (1996). Radiation effects on MHD free convection flow of a gas past a semi-infinite vertical plate. Int. J. Num. Methods Heat Fluid Flow 6, 77-83.

Vafai, K. (2005). Handbook of porous media, second edition, Taylor and Francis Group, USA. 